\title{
Radial pulsation among $\delta$ Sct stars
}

\author{
E. Rodríguez, A. Rolland, P. López de Coca, S. Martín \\ Instituto de Astrofísica de Andalucía, CSIC, Apdo. 3004, 18080 \\ Granada, Spain
}

\begin{abstract}
It has been pointed out by earlier authors that radial pulsation seems to be the only one type present in high amplitude $\delta$ Sct stars, at least for monoperiodic stars. In order to confirm or not this point, we have collected all the multicolour data available for these stars in the Strömgren and Johnson photometric systems. Then, the type of pulsation has been analysed on the basis of the phase shifts and amplitude ratios between observed light and colour variations. The results indicate that all the stars analysed are radial pulsators.
\end{abstract}

It has been pointed by earlier authors that two clearly different groups of pulsators seems to coexist among the $\delta$ Sct stars. First, variables with low amplitudes of pulsation (visual amplitudes smaller than roughly $0 .^{m} 1$ ). The majority of these variables show complex pulsation frequency spectra where a number of simultaneously excited nonradial (and radial) modes are present. Second, variables with high amplitudes of pulsation (visual amplitudes greater than roughly $0 .^{m} 3$ ). In most of them only one or two frequencies appear excited, and both single and double mode stars seem to show radial pulsation.

In all these double mode high amplitude stars, radial pulsation has been found from comparison between observed and theoretical period ratios. Moreover, the method based on "amplitude ratios versus phase shifts" between different filters, in $u v b y$ photometry, was applied by earlier authors to a large sample of monoperiodic high amplitude $\delta$ Sct and SX Phe stars leading to radial pulsation for all the variables analysed. A similar method has also been shown in UBV photometry. Thus, radial pulsation seems to be the only one type present in high amplitude $\delta$ Sct stars, at least for mono-periodic and double mode pulsators.

In order to confirm or not this point for mono-periodic stars, we have collected all the simultaneous or nearly simultaneous multicolour data available for these stars in the Strömgren and Johnson photometric systems. The data have been considered for Population I and also Population II (SX Phe type) stars. Then, the type of pulsation has been analysed on the basis of these phase shifts and amplitude ratios between observed light and colour variations. Table 1 lists the stars analysed with their periods and full visual amplitudes. In all cases, the results lead to radial pulsation according to the corresponding diagrams of "amplitude ratios versus phase shifts". In addition to mono-periodic variables, some double mode stars (AE UMa, RV Ari, BP Peg, V1719 Cyg and SX Phe) were considered and radial pulsation was also found in good agreement with their period ratios. It is also true for the two first modes of AI Vel. Radial pulsation was also found for the three mono-periodic stars of medium amplitude $\left(0 .{ }^{m} 1 \leq \Delta \mathrm{V}<0 .{ }^{m} 3\right)$ considered: V1162 Ori, $\rho$ Pup and V393 Car. 
Table 1. Stars analysed.

\begin{tabular}{|c|c|c|c|c|c|c|c|}
\hline Star & $\begin{array}{l}\text { Perjod } \\
\text { (days) }\end{array}$ & $\underset{(\mathrm{mag})}{\Delta V}$ & source & Star & $\begin{array}{l}\text { Period } \\
\text { (days) }\end{array}$ & $\underset{\text { (mag) }}{\Delta V}$ & source \\
\hline Pop. I & & & & Pop. I & & & \\
\hline ZZ Mic & 0.0672 & 0.35 & $23,24,25$ & V567 Oph & 0.1495 & 0.33 & 6,7 \\
\hline GP And & 0.0787 & 0.52 & 1 & CW Ser & 0.1892 & 0.43 & 15,16 \\
\hline V1162 Ori & 0.0787 & 0.20 & 6 & V798 Cyg & 0.1948 & 0.46 & 4 \\
\hline KU Cen & 0.0800 & 0.47 & 6 & BS Aqr & 0.1978 & 0.44 & 10,11 \\
\hline AE UMa(F) & 0.0860 & 0.44 & 1 & RY Lep & 0.2254 & 0.35 & $i$ \\
\hline EH Lib & 0.0884 & 0.50 & $17,18,19,20,21,22$ & TV Lyn & 0.2407 & 0.44 & 1 \\
\hline RV Ari(F) & 0.0931 & 0.43 & 2 & DE Lac & 0.2537 & 0.32 & 12,13 \\
\hline BE Lyn & 0.0959 & 0.39 & 1 & $\mathrm{DH} \mathrm{Peg}_{\mathrm{g}}$ & 0.2555 & 0.50 & 14 \\
\hline YZ Boo & 0.1041 & 0.42 & $26,27,28,29$ & UY Cam & 0.2670 & 0.34 & 1 \\
\hline BP $\operatorname{Peg}(\mathrm{F})$ & 0.1095 & 0.41 & 1 & V1719 Cyg(1) & 0.2673 & 0.31 & 4 \\
\hline AI Vel(F) & 0.1116 & 0.67 & 3 & V1719 Cyg(2) & 0.2138 & & 4 \\
\hline AI Vel(1) & 0.0862 & & 3 & Pop. II & & & \\
\hline SZ Lyn & 0.1205 & 0.51 & $29,34,35,36,37,38$ & SXPhe(F) & 0.0550 & 0.41 & $T$ \\
\hline AD CMi & 0.1230 & 0.30 & 1 & SX Phe(1) & 0.0428 & & 1 \\
\hline$\rho$ Pup & 0.1409 & 0.09 & 8,9 & KZ Hya & 0.0595 & 0.80 & 1 \\
\hline V393 Car & 0.1413 & 0.19 & 5 & CY Aqr & 0.0610 & 0.71 & 1 \\
\hline RS Gru & 0.1470 & 0.56 & 1 & DY Peg & 0.0729 & 0.54 & 1 \\
\hline DY Her & 0.1486 & 0.51 & $30,31,32,33$ & $\mathrm{XX} \mathrm{Cyg}$ & 0.1349 & 0.80 & 1 \\
\hline
\end{tabular}

1 Garrido et al. 1990, A\&A 234, 262

2 Rodríguez et al. 1992, A\&AS 93, 189

3 Watson R.D. 1988, Ap\&SS 140, 255

4 Poretti et al. 1988, A\&A 199, 191

5 Helt B.E. 1984, A\&AS 56, 457

6 Poretti et al. 1990, A\&A 228, 350

7 Powell et al. 1990, PASP 102, 1131

8 Balona et al. 1983, South African Astr. Obs. Circ. 7, 19

9 Dravins et al. 1977, A\&A 54, 381

10 Elst E.W. 1976, A\&AS 23, 419

11 Meylan et al. 1986, A\&AS 64, 25

12 Fitch et al. 1966, Comm. Lunar Planet. Observ. 71, 3

13 Paczynski B. 1965, Acta Astron. 15, 115

14 Tifft W.G. 1964, ApJ 139, 451

15 Gieren et al. 1975, Inf. Bull. Var. Stars No. 1055

16 Bookmeyer et al. 1977, Rev. Mex. Astron. Astrof. 2, 235

17 Joner M.D. 1986, PASP 98, 651

18 Broglia et al. 1977, A\&AS 29, 321

19 Terzan et al. 1974 , A\&AS 16,155

20 Garrido et al. 1979, A\&AS 36, 51

21 Mahdy et al. 1980 , Comm. Konkoly Obs. No. 74

22 Karetnikov et al. 1979, Perem. Zvezd. 21, 199

23 Churms et al. 1961, Observatory 920, 25

24 Balona et al. 1978, MNRAS 184, 11

25 Chambliss C.R. 1971, ApJ 165, 365

26 Szeidl et al. 1981, Comm. Konkoly Obs. No. 75

27 Broglia et al. 1957, Cont. Ob. Ast. Mil.-Mer. No. 102

28 Joner et al. 1983, PASP 95, 433

29 Langford W.R. 1976, Ph. D. Thesis Brigham Univ.

30 Breger et al. 1978, PASP 90, 754

31 Hardie et al, 1961, ApJ 133, 71

32 Spinrad H. 1959, ApJ 130, 539

33 Broglia et al. 1955, Cont. Ob. Ast. Mil.-Mer. No. 66

34 Soliman et al. 1986 , Comm. Konkoly Obs. No. 88

35 Broglia P. 1963, Cont. Ob. Ast. Mil.-Mer. No. 222

36 Barnes et al. 1975, AJ 80, 48

37 Duerbeck H.W. 1976, Inf. Bull. Var. Stars No. 1971

38 Szeidl B. 1983 , Comm. Konkoly Obs. No. 84 